\title{
GROWTH OF A PEANUT-SHAPED BULGE VIA RESONANT TRAPPING OF STELLAR ORBITS IN THE VERTICAL INNER LINDBLAD RESONANCES
}

\author{
A. C. Quillen ${ }^{1}$ \\ Department of Physics and Astronomy, University of Rochester, Rochester, NY 14627; aquillen@pas.rochester.edu \\ Received 2002 March 18; accepted 2002 May 9
}

\begin{abstract}
We present a simple resonant Hamiltonian model for the vertical response of a stellar disk to the growth of a bar perturbation. As a bar perturbation grows, stars become trapped in vertical inner Lindblad resonances and are lifted into higher amplitude orbits. The vertical structure of a boxy and peanut-shaped bulge as a function of radius and azimuthal angle in the galaxy plane can be predicted from the strength and speed of the bar perturbation and the derivatives of the gravitational potential. This model predicts that stars on the outer side of the resonance are lifted higher than stars on the inner side, offering an explanation for the sharp outer edge of the boxy/peanut.
\end{abstract}

Key words: galaxies: kinematics and dynamics

\section{INTRODUCTION}

The existence of spiral galaxy bulges with boxy or peanut shapes has been recognized for many years through the study of edge-on galaxies (e.g., Burbridge \& Burbridge 1959; de Vaucouleurs 1974). Recent work has found that edge-on galaxies with prominent boxy or peanut-shaped bulges, in most cases, show kinematic evidence for noncircular motions associated with a bar, whereas galaxies lacking boxy or peanut-shaped bulges do not show kinematic evidence for a bar (Bureau \& Freeman 1999; Merrifield \& Kuijken 1999). A few cases exist where highly inclined systems exhibit both bars and peanuts (e.g., NGC 7582, Quillen et al. 1997 and NGC 4442, Bettoni \& Galletta 1994). The link between a galactic bar and a boxy/peanutshaped bulge has been firmly established.

Mechanisms proposed to explain the formation of boxy and peanut-shaped structures include accretion of small satellite galaxies (Binney \& Petrou 1985), bar buckling via the fire-hose-type instability (Toomre 1966; Raha et al. 1991; Merritt \& Sellwood 1994; Fridman \& Polyachenko 1984), and resonant heating (Pfenniger 1984, 1985; Combes et al. 1990).

The fire-hose (bar-buckling) instability is a global timedependent instability that relies on the centrifugal force caused by highly eccentric orbits in the plane caused by the bar and is damped via a dissipative process similar to Landau damping (Fridman \& Polyachenko 1984). The resonant heating model has been inspired by the study and classification of orbit families in three-dimensional systems (Pfenniger \& Friedli 1991) and through the phenomena displayed in $N$-body simulations (Combes et al. 1990).

No study has yet considered the evolution of threedimensional stellar orbits in a barred system that is varying with time. In this paper, we investigate the possibility that the growth of the bar itself traps particles in the vertical inner Lindblad resonances (ILRs). We develop a simple Hamiltonian analytical model for these resonances and use it to predict the vertical distribution of a bar following the growth of the bar. Our approach of considering resonance trapping is similar to that explored by Sridhar \& Touma

\footnotetext{
${ }^{1}$ Visitor, Department of Physics, Technion, Israel Institute of Technology.
}

(1996) for disk heating. However, their model is for the general case of a growing axisymmetric disk, whereas our model applies specifically to a growing bar perturbation.

\section{DYNAMICAL MODEL}

To exhibit resonant phenomena, Contopoulos (1975) showed that the Hamiltonian must be expanded to at least third order in the epicyclic approximation. When this is done, there are higher order cross terms in the action variables, and the model will exhibit phenomenology such as the bifurcation of orbit families seen in characteristic diagrams, which are created through numerical integration of orbits.

We first consider the Hamiltonian lacking nonaxisymmetric or time-dependent perturbations:

$$
H_{0}\left(L, p_{r}, p_{z} ; \theta, r, z\right)=\frac{L^{2}}{2 r^{2}}+\frac{p_{r}^{2}}{2}+\frac{p_{z}^{2}}{2}+V_{0}(r, z),
$$

where $V_{0}$ is the gravitational potential, which is assumed to be axisymmetric, and $L, p_{r}$, and $p_{z}$ are the momenta conjugate to the cylindrical coordinates $\theta, r$, and $z$. For the twodimensional problem restricted to the Galactic plane $(z=0$, $p_{z}=0$ ), Contopoulos (1975) showed how to put the Hamiltonian in the form

$$
K_{0}\left(I_{1}, I_{2} ; \theta_{1}, \theta_{2}\right)=h+\kappa I_{1}+\Omega I_{2}+a I_{1}^{2}+2 b I_{1} I_{2}+c I_{2}^{2} \cdots
$$

in a third-order epicyclic approximation. The action variables $I_{1}=1 / 2 \pi \int \dot{r} d r$ and $I_{2}=J_{0}-J_{c}$ are integrals of motion. $J_{0}$ is the particle's angular momentum, and $J_{c}$ is the angular momentum of a particle in a circular orbit at a radius $r_{c}$, which is the radius of a circular orbit with energy $h$. The variable $\theta_{1}$ is the epicyclic angle, and $\theta_{2}$ is the azimuth of the epicyclic center. Here $\theta_{1}$ and $\theta_{2}$ are the angle variables conjugate to $I_{1}$ and $I_{2}$. The variable $\kappa$ is the epicyclic frequency, and $\Omega$ is the angular rotation rate; both are evaluated at $r=r_{c}, z=0$.

To extend the theory into the third dimension, we must add additional terms to the restricted Hamiltonian that depend upon $z$ :

$$
H_{0}=K_{0}+H_{0 z}
$$


We expand the potential $V_{0}$ about $z$ and $r_{c}$, defining the variables

$$
\begin{aligned}
\nu^{2} & =\partial^{2} V_{0} / \partial z^{2}, \\
\lambda & =\partial^{4} V_{0} / \partial z^{4},
\end{aligned}
$$

which are evaluated at $z=0, r=r_{c}$. Inserting this into the full Hamiltonian, we find

$$
\begin{aligned}
H_{0 z} & \left(L, p_{r}, p_{z} ; \theta, r, z\right)=\frac{1}{2}\left(p_{z}^{2}+\nu^{2} z^{2}\right)+\frac{\lambda z^{4}}{4 !} \\
& +\frac{\nu^{2} z^{2}}{2}\left[r_{c} \Omega^{2}\left(r-r_{c}\right)+\frac{1}{2}\left(\kappa^{2}-3 \Omega^{2}\right)\left(r-r_{c}\right)^{2}\right],
\end{aligned}
$$

where we have made use of the relations $\Omega^{2}=(1 / r)\left(\partial V_{0} / \partial r\right)$ and $\kappa^{2}=\left(\partial^{2} V_{0} / \partial r^{2}\right)+3 \Omega^{2}$, and both $\Omega$ and $\kappa$ are evaluated at $r=r_{c}, z=0$.

For this Hamiltonian, following Contopoulos (1975), we can choose a third action variable $I_{3}$ to represent the amplitude of vertical oscillations:

$$
\begin{aligned}
z & \approx\left(2 I_{3} / \nu\right)^{1 / 2} \cos \theta_{3}, \\
d z / d t & \approx-\left(2 I_{3} \nu\right)^{1 / 2} \sin \theta_{3},
\end{aligned}
$$

where $\nu$ is the vertical oscillation frequency, and $\theta_{3}$ is the angle associated with the vertical oscillations. The above expressions are given to first order in $I_{3}^{1 / 2}$ and require higher order terms to be exact.

After a couple of canonical transformations (see Appendix), the Hamiltonian to second order in $I_{3}^{1 / 2}$ takes the form

$$
H_{0 z}\left(I_{1}, I_{2}, I_{3} ; \theta_{1}, \theta_{2}, \theta_{3}\right)=\nu I_{3}+\frac{\lambda I_{3}^{2}}{32 \nu^{2}}+\left(\kappa^{2}-3 \Omega^{2}\right) \frac{\nu I_{1} I_{3}}{4 \kappa} .
$$

Since this is independent of the angles $\theta_{1}, \theta_{2}$, and $\theta_{3}$, the momenta $I_{1}, I_{2}$, and $I_{3}$ are conserved quantities and we say we have put the nonperturbed Hamiltonian (eq. [5]) in action angle form.

\subsection{Perturbation}

We now consider the form of the perturbation of the gravitational potential caused by a bar. In the plane of the galaxy, we can expand the gravitational potential in terms of Fourier components

$$
V_{1}(r, \theta, z=0)=\sum_{m} A_{m} \cos \left[m\left(\theta-\Omega_{b} t\right)\right]
$$

(e.g., see Quillen, Frogel, \& Gonzalez 1994). The strongest term due to the bar should be the $m=2$ term with $A_{2}<0$, so that the bar major axis lies along $\theta-\Omega_{b} t=0, \pi$. For small $z$, we assume that the potential can be expanded, so that we can write for each $m$ Fourier component

$$
V_{1 m}(r, \theta, z)=A_{m} \cos \left[m\left(\theta-\Omega_{b} t\right)\right]\left(1-\frac{z^{2}}{2 h_{1}^{2}}\right),
$$

where $h_{1}$ is the scale height of the perturbation. We also assume that $A_{m}$ and $h_{1}$ vary only weakly with radius. Since $z^{2} \approx\left(2 I_{3} / \nu\right) \cos ^{2} \theta_{3}$, we can rewrite the perturbation components to second order in $I_{3}^{1 / 2}$ as

$$
V_{1 m}\left(I_{3}, \theta_{3} ; I_{2}, \theta_{2}\right)=\frac{-A_{m} I_{3}}{4 h_{1}^{2} \nu} \cos \left[m\left(\theta_{2}-\Omega_{b} t\right) \pm 2 \theta_{3}\right]+\cdots .
$$

We focus on the particular resonance that we expect is important and ignore all the other terms:

$$
\begin{aligned}
H\left(I_{2}, I_{3} ; \theta_{2}, \theta_{3}\right) & =\nu I_{3}+\frac{\lambda}{32 \nu^{2}} I_{3}^{2}+\Omega I_{2} \\
+ & I_{3} \frac{-A_{m}}{4 h_{1}^{2} \nu} \cos \left[m\left(\theta_{2}-\Omega_{b} t\right)-2 \theta_{3}\right]+\cdots .
\end{aligned}
$$

To go into a coordinate system with an angle that librates when a particle is trapped in the resonance, we do a canonical transformation with the following generating function:

$F_{2}\left(J_{3}, J_{2} ; \theta_{3}, \theta_{2}\right)=J_{2} \theta_{2}-\left(J_{3} / 2\right)\left[m\left(\theta_{2}-\Omega_{b} t\right)-2 \theta_{3}\right]$,

so that our resonant angle and new momenta,

$$
\begin{aligned}
\phi & =\theta_{3}-(m / 2)\left(\theta_{2}-\Omega_{b} t\right), \\
I_{2} & =J_{2}-J_{3}(m / 2), \\
I_{3} & =J_{3},
\end{aligned}
$$

and $\theta_{2}$ is unchanged. Our generating function is time dependent: $\partial F_{2} / \partial t=(m / 2) \Omega_{b} J_{3}$. Following this canonical transformation, our Hamiltonian becomes

$$
\begin{aligned}
H\left(J_{2}, J_{3} ; \theta_{2}, \phi\right)= & {\left[\nu-\frac{m}{2}\left(\Omega-\Omega_{b}\right)\right] J_{3}+\frac{\lambda}{32 \nu^{2}} J_{3}^{2} } \\
& +J_{3} \frac{-A_{m}}{4 h_{1}^{2} \nu} \cos 2 \phi+\Omega J_{2}+\cdots .
\end{aligned}
$$

The motion in the two different degrees of freedom are now decoupled, so we can neglect $J_{2}$ and write the Hamiltonian in the simpler form

$$
H\left(J_{3} ; \phi\right)=\nu^{\prime} J_{3}+a J_{3}^{2}-\epsilon J_{3} \cos 2 \phi,
$$

where

$$
\begin{aligned}
\nu^{\prime} & \equiv \nu-(m / 2)\left(\Omega-\Omega_{b}\right), \\
a & \equiv \lambda / 32 \nu^{2}, \\
\epsilon & \equiv A_{m} / 4 h_{1}^{2} \nu .
\end{aligned}
$$

Equation (15) has a resonant term proportional to $J_{3}$ instead of proportional to $J_{3}^{1 / 2}$, as was the case for the inner Lindblad resonance considered by Contopoulos (1975) and for $e$-e' orbit-orbit resonances commonly studied in celestial mechanics (Murray \& Dermott 1999). However, the Hamiltonian is similar to that of $J+2: J$ orbital resonances studied by Borderies \& Goldreich (1984). Orbits captured in the $2: 1$ vertical ILR were seen in the numerical simulations of Combes et al. (1990), but they expected the resonance to be first order in $J_{3}^{1 / 2}$ rather than second order as we have found here. By symmetry, the gravitational potential when expanded should not contain terms proportional to $z$, and so terms $\propto J_{3}^{1 / 2}$, unless we consider a global instability such as the fire-hose bar-buckling instability. Indeed, Pfenniger \& Friedli (1991) found that a temporal $z$-symmetry breaking (due to a collective effect) would make the resonance first order. Friedli \& Pfenniger (1990) reported that $N$-body simulations that forced $z$-symmetry did grow boxy/peanut shaped bulges but more slowly than those that did not force the symmetry.

Unless the galaxy is extremely thin and the bulge small, we expect that the $2: 1$ vertical ILR will occur in the inner region of the bar (Pfenniger \& Friedli 1991; Combes et al. 1990). It is also possible that the $1: 1$ vertical ILR will be present. The 2:1 vertical ILR produces banana-shaped 
orbits, referred to as BAN in the characteristic diagrams of Pfenniger \& Friedli (1991), and the 1:1 vertical ILR produces titled orbits that are referred to as anomalous by Pfenniger \& Friedli (1991). If the galaxy is very thin, it is also possible that there will be orbits associated with the $4: 1$ vertical ILR (Pfenniger 1985).

If we concentrate on the $2: 1$ vertical ILR, then the perturbation term from the bar that is relevant is the $m=4$ Fourier term; if we focus on the $1: 1$ vertical ILR then the $m=2$ term is relevant. It has long been known that bars exhibit significant $m=4$ Fourier components and contain boxy or square contours when projected onto the plane of the galaxy (Athanassoula 1990). From Figure 4 by Quillen et al. (1994), we estimate that in the $m=4$ Fourier component of the barred galaxy NGC 4314, the potential peaks at $A_{4} \sim-0.04$ in units of $v_{c}^{2}$ and at $A_{2} \sim-0.16$ in the same units. We can use these as typical values for the potential perturbations in a barred galaxy.

We now transform equation (15) so that our coefficients are unitless. We put length in units of $r_{\text {res }}$, the center of the resonance where $\nu^{\prime}=0$, and time in units of $1 / \nu$. Our action variables are in units of $r_{\text {res }}^{2} \nu$, so we define $j_{3} \equiv J_{3} / r_{\text {res }}^{2} \nu$. We divide the entire Hamiltonian by $r_{\text {res }}^{2} \nu^{2}$, so that our new Hamiltonian becomes

$$
h\left(j_{3}, \phi\right) /\left(a r_{\text {res }}^{2}\right)=j_{3}^{2}+\bar{\nu} j_{3}-\bar{\epsilon} j_{3} \cos 2 \phi,
$$

where

$$
\begin{aligned}
& \bar{\nu} \equiv \frac{32 \nu^{\prime} \nu}{r_{\mathrm{res}}^{2} \lambda} \sim \frac{-32 \nu^{\prime}}{\nu} \frac{h_{1}^{2}}{r_{\mathrm{res}}^{2}}, \\
& \bar{\epsilon} \equiv \frac{8 A_{m}}{r_{\mathrm{res}}^{2} h_{1}^{2} \lambda} \sim \frac{-8 A_{m}}{v_{c}^{2}} \frac{v_{c}^{2}}{\nu^{2} r_{\mathrm{res}}^{2}}
\end{aligned}
$$

and we have assumed that the vertical form of the potential can be approximated by $\lambda \sim-\nu^{2} / h_{1}^{2}$. The value of $\lambda$ should be negative because the vertical oscillations are expected to be slower for particles traveling well above the galactic plane.

For a bar with a peak, $A_{4} / v_{c}^{2} \sim-0.04$, as estimated from NGC 4314. We find $\nu r_{\text {res }} \sim 2 \Omega r_{\text {res }} \sim 2 v_{c}$ at the $2: 1$ resonance, and $\nu r_{\text {res }} \sim v_{c}$ at the $1: 1$ resonance. We estimate that $\bar{\epsilon}$ peaks at $\sim 0.08$ for both resonances because $A_{2} \sim 4 A_{4}$. Barred galaxies have an observed ratio of bar length to peanut length of $2.7 \pm 0.3$ (Lütticke, Dettmar, \& Pohlen 2000). Measurements of the ratio of bar length to thickness give a typical value of $\sim 10$ (Sellwood \& Wilkinson 1993), with the measurement of Lütticke et al. (2000) being on the high end at $14 \pm 4$ and the Milky Way bar likely to be lower than the typical value. Using these typical values, we estimate that $h_{1} / r_{\text {res }} \sim 0.3$ :

$$
\begin{aligned}
& \bar{\nu} \sim-3 \frac{\nu^{\prime}}{\nu}\left(\frac{h_{1} / r_{\text {res }}}{0.3}\right)^{2}, \\
& \bar{\epsilon} \sim 0.08\left(\frac{-A_{m} / v_{c}^{2}}{0.04}\right)\left(\frac{2}{\nu r_{\text {res }} / v_{c}}\right)^{2} .
\end{aligned}
$$

We expect the resonance to be important in the region where $\bar{\nu}=0 \rightarrow \bar{\epsilon}$ or across a range $d r$ :

$$
\frac{d r \nu_{, r}^{\prime}}{\nu}=\frac{-A_{m}}{4 \nu^{2} h_{1}^{2}},
$$

where the subscript " ${ }_{, r} "$ denotes a derivative with respect to radius. If $\bar{\nu}$ and $\nu^{\prime}$ varies slowly with radius, then the resonance can be felt over a significant range of radius.

Given the definition of our variables, we estimate that the expectation value

$$
\left\langle z^{2}\right\rangle \sim\left\langle j_{3}\right\rangle r_{\text {res }}^{2}
$$

From Figure 1, we infer that when $\bar{\nu} / \bar{\epsilon}=-1$, the minimum value of vertical oscillations occurs for the fixed points at $j_{3}=1$. We therefore expect the maximum height of the peanut to be at $\bar{\nu} / \bar{\epsilon}=-1$, where $\left\langle j_{3}\right\rangle \sim \bar{\epsilon}$. For $\bar{\epsilon} \sim 0.08$, this implies a peanut width of order $2 \sqrt{\bar{\epsilon}} r_{\text {res }} \sim 0.6 r_{\text {res }}$, where we gain the factor of 2 when we consider orbits of both signs $(\phi=0$ and $\phi=\pi)$. This is sufficiently large to explain the observed vertical extent of boxy/peanut shaped bulges.

\subsection{Structure of the Resonance}

As shown by Borderies \& Goldreich (1984), the resonance contains three separate regions. To show the separate regions, we use a coordinate system $x=\left(2 j_{3}\right)^{1 / 2} \cos \phi$ and $y=\left(2 j_{3}\right)^{1 / 2} \sin \phi$. Contours of constant $h$ (eq. [17]) are shown in Figure 1. For $\bar{\nu} / \bar{\epsilon}<-1$, three fixed points exist; for $-1<\bar{\nu} / \bar{\epsilon}<1$, two fixed points exist; and for $\bar{\nu} / \bar{\epsilon}>1$, only one fixed point exists. Orbits circulating around $\phi=0$ correspond to banana-shaped orbits with positive $z$ at the ends of the bar, and orbits correspond to banana-shaped orbits with negative $z$ at the ends of the bar. As shown in Figure $8 b$ of Pfenniger \& Friedli (1991), banana-shaped orbits appear on the characteristic diagram at a particular value of the Hamiltonian, consistent with them appearing at a particular value of $\bar{\nu} / \bar{\epsilon}$. For orbits circulating around the origin, the resonant angle, $\phi$, circulates; referred to as oscillation as opposed to libration in analogy with a pendulum.

We consider the signs of each term in equations (15) and (17) $\nu>0$, but we expect that $\lambda, A_{4}, A_{2}<0$. Consequently, $a<0$ and $\epsilon, \bar{\epsilon}>0$, and $\bar{\nu}$ has the opposite sign of $\nu^{\prime}$. Because $\nu$ and $\Omega$ increase at small radii, we expect $\nu^{\prime}>0$ and $\bar{\nu}<0$ for $r>r_{\text {res }}$. We see from Figure 1 that near $\bar{\nu}=-\bar{\epsilon}$ no orbits exist with low values of $j_{3}$. This implies that all orbits will rise out of the plane of the galaxy. As $\bar{\nu}$ decreases the minimum size of the vertical oscillations increases until $\bar{\nu} / \bar{\epsilon}<-1$, then orbits with small amplitude vertical oscillations appear again. The vertical extent of the galaxy should increase until a particular radius and then drop abruptly (see Fig. 2). This would naturally correspond to a bow tie or peanut shape when viewed edge-on.

\subsection{Growing the Bar}

To explore the simple model given in equation (17), we integrate particle trajectories assuming that the perturbation is growing. In other words, we assume that $\bar{\epsilon}$ begins at zero and reaches a maximum value a few rotation periods later. We begin the integrations with a particle distribution $p\left(j_{3}\right) \propto \exp \left(-j_{3}^{2} / 2 \sigma^{2}\right)$ and with an angle $\phi$ randomly chosen between 0 and $2 \pi$. Four thousand particles were integrated using equation (17) for the simulations shown in Figures 36 . The growth time for the perturbation is given in periods, where $P=(2 \pi / \nu)|a| r_{\text {res. }}^{2}$ Since $|a| r_{\text {res }}^{2} \sim\left(r_{\text {res }}^{2} / h_{1}^{2} 32\right) \sim 1 / 3, P$ is about one-third the bar growth time in units of the vertical oscillation period.

In the adiabatic limit, the capture probability of a region of phase space depends upon the ratio between the rate that phase-space volume is swept across the separatrix bounding this region divided by the rate that phase-space volume is 


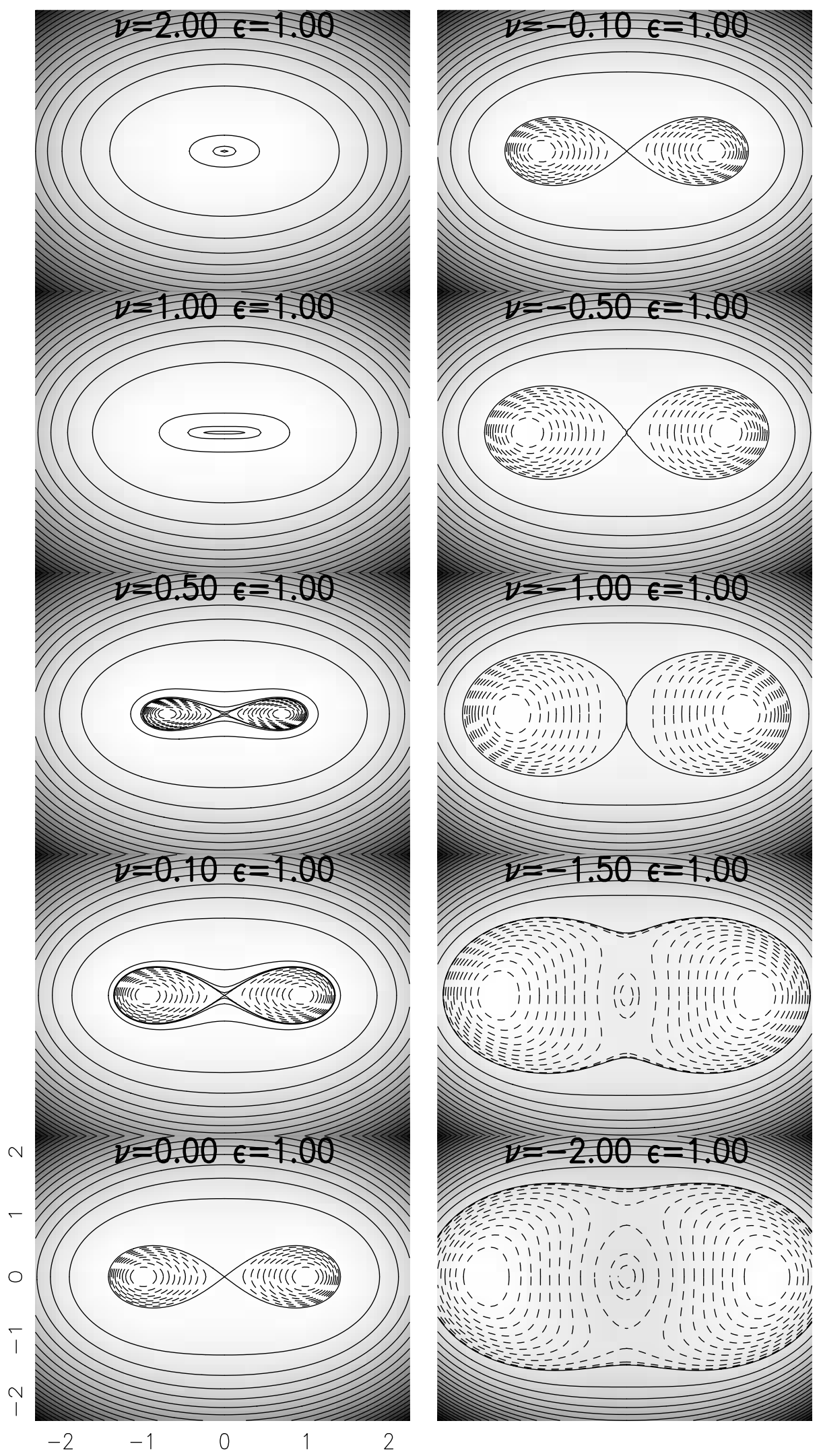

Fig. 1. Contour levels of the Hamiltonian in eq. (17) have been plotted where $x=\left(2 j_{3}\right)^{1 / 2} \cos \phi$ and $y=\left(2 j_{3}\right)^{1 / 2}$ sin $\phi$. Only one resonance region exists for $\bar{\nu} / \bar{\epsilon}>1$, two exist for $-1<\bar{\nu} / \bar{\epsilon}<1$, and three for $\bar{\nu} / \bar{\epsilon}<-1$. The resonance bifurcates at $\bar{\nu} / \bar{\epsilon}= \pm 1$ giving banana-shaped orbits at $\phi=0$ and upside-down banana-shaped orbits at $\phi=\pi$. The bar strength sets $\bar{\epsilon}$, and $\bar{\nu}$ varies with radius from the center of the resonance where $\bar{\nu}=0$. Between $\bar{\nu} / \bar{\epsilon}=-1 \rightarrow 1$, no small $j_{3}$ circulating orbits exist. We can think of the progression of resonance sizes between $\bar{\nu} / \bar{\epsilon} \sim-1 \rightarrow 1$ as describing the radial variation of the width of the peanut. We expect maximum vertical amplitudes at $\bar{\nu} / \epsilon \sim-1$, which is on the outer, larger radius side of the resonance. As the bar grows, phase space moves downward from plot to plot, and particles are trapped and then lifted upward. Likewise, if the bar slows down, the same thing will happen. 


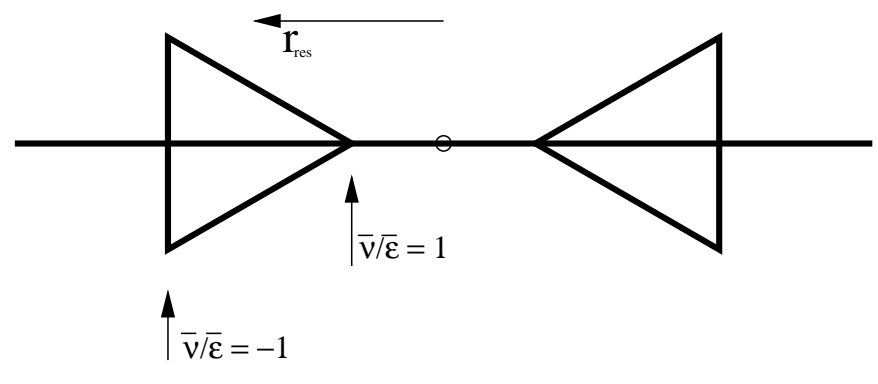

FIG. 2.-Cartoon of the edge-on galaxy labeling the approximate location of the peanut in terms of the values of $\bar{\nu} / \bar{\epsilon}$.

swept across the entire separatrix bounding the entire resonance (Henrard 1982; Borderies \& Goldreich 1984). For $\bar{\nu}<0$, phase space initially looks like the panel on the bottom right of Figure 1 and then moves upward on this figure. When the separatrix touches the particle distribution centered at the origin, particles are captured into librating regions near $\phi=0$ and $\pi$ (see Figs. 3-4). For $\bar{\nu}>0$, phase space initially looks like the panel on the top left of Figure 1 and then moves downward on this figure. When $\bar{\nu} \sim \bar{\epsilon}$, particles at low $j_{3}$ are captured into the librating regions around $\phi=0, \pi$. Figures 3-4 illustrate that smoother particle distributions are achieved when the growth rate is faster (shorter number of periods $P$ ), as we would expect when the variation is no longer adiabatic and when the initial momentum distribution is wider (larger $\sigma$ ).

Given a distribution in $j_{3}$ and $\phi$ resulting from the growth of the perturbation, we can predict the vertical distribution as a function of azimuthal angle in the plane of the galaxy. For a value of the resonant angle $\phi$ and an azimuthal angle $\theta-\Omega_{b} t$ in the plane of the galaxy in the frame rotating with the bar, $\theta_{3}$ is determined via equation (13), and $z$ is then determined from the definition of our action angle variables (eq. [6]). In Figure 5, we show vertical distributions for the action angle distributions shown in Figures 3 and $4 b$ for $m=2$, and in Figure 6 we show the equivalent but for $m=4$.

We see from Figures 5 and 6 that the vertical height reaches a maximum near $\bar{\nu} / \bar{\epsilon} \sim-1$, as we expected from the form of the resonance. The height increases slowly between $\bar{\nu} / \bar{\epsilon} \sim 1$ and -1 and then drops swiftly, confirming conceptually our bow-tie picture shown in Figure 2. For $m=2$ (shown in Fig. 5), corresponding to the $1: 1$ vertical ILR, the major axis of the bar becomes bulbous, as would be expected from a sum of anomalous orbits of both orientations (shown in Fig. 7). For $m=4$ (shown in Fig. 5), corresponding to the $2: 1$ vertical ILR, both the major and minor axes of the bar have increased vertical heights. Again, this is expected from a sum of banana-shaped orbits of both orientations (see Fig. 7).

Observations of peanut-shaped galaxies where both the bar and the peanut are observed (e.g., Quillen et al. 1997) suggest that the ends of the peanut are aligned with the bar. As a result, it could be that the $1: 1$ resonance orbit family is more likely to explain the bulbous ends of boxy/peanut shaped bulges. However, when the radial oscillations of the galaxy are taken into account, the banana-shaped orbits may still be viable, particularly if the extent of their vertical oscillation is smaller along the minor axis than it is along the major axis.
We remind the reader that in $\S 2.1$ we dropped the last term in equation (7), which is proportional to $I_{1} I_{3}$. As a bar grows, the epicyclic amplitude $I_{1}$ of the stars will grow. This has the effect of shifting the location of the vertical resonance so that $\nu^{\prime}$ becomes

$$
\nu^{\prime}=\nu-\frac{m}{2}\left(\Omega-\Omega_{b}\right)+\left(\kappa^{2}-3 \Omega^{2}\right) \frac{\nu I_{1}}{4 \kappa} .
$$

For a flat rotation curve, $\kappa^{2}=2 \Omega^{2}$, so we expect that $\nu^{\prime}$ will be more negative than expected if this additional term is neglected. This has the affect of moving the resonance outward in radius. One reason that the $2: 1$ ILR resonance was considered a logical choice for exciting the peanut is that when the galaxy is thin, the $1: 1$ may only occur at extremely small radii, well inside the galaxy bulge. However, the estimates for the resonance locations were made assuming $I_{1} \sim 0$ (e.g., Pfenniger \& Norman 1990). The point here is that the growth of the epicyclic amplitude will shift the location of the vertical resonances outward, so that the lower order resonances may manifest at larger radii than previously considered. It is possible that the growth of the bar causes the vertical resonances to sweep through the inner part of the galaxy.

\section{SUMMARY AND DISCUSSION}

We have presented a simple Hamiltonian dynamical model for the vertical ILR in the presence of a bar perturbation. The Hamiltonian model is similar to that introduced by Contopoulos (1975) to describe the 2:1 Lindblad resonances in the plane of the galaxy but uses the vertical action angle variables. Using this model, we consider the growth of a boxy/peanut shaped bulge via resonance trapping in the vertical ILR caused by the growth of the bar. Following the growth of the bar, our model predicts that the height of the peanut increases as a function of radius until it reaches a maximum at the outermost radius that is set by the condition $\bar{\nu} / \bar{\epsilon}=-1$. Our model naturally accounts for the sharp outer edge of observed boxy/peanut shaped bulges. The maximum total vertical width of the peanut is of order $\sim 2 \sqrt{\bar{\epsilon}} r_{\text {res }}$, which we estimate for a typical bar could be about $\frac{2}{3} r_{\text {res }}$, where $r_{\text {res }}$ is the radial location of the resonance. This model is therefore capable of accounting for the observed widths of boxy/peanut shaped bulges.

The scenario explored here suggests that the boxy/peanut shaped bulge is grown at the same time as the bar forms. We predict that recently formed bars should manifest strong boxy/peanut shaped bulges, and barred galaxies should never be found without boxy/peanut shaped bulges. If the boxy/peanut shapes take nearly a Hubble time to grow, as found in the $N$-body simulations of Combes et al. (1990), then there should be barred galaxies that lack boxy/peanut shaped bulges. However, Bureau \& Freeman (1999) considered a control sample of edge-on galaxies lacking boxy/peanut bulges. No examples of barred galaxies that lack a boxy/peanut shaped bulge have yet been identified. Pfenniger \& Friedli (1991) found that the boxy/peanut only required 3-4 bar growth periods to grow, and this could be significantly shorter, only a few gigayears. If this timescale were more appropriate, then the lack of barred galaxies that do not display boxy/peanut shaped bulges is less of a problem. 


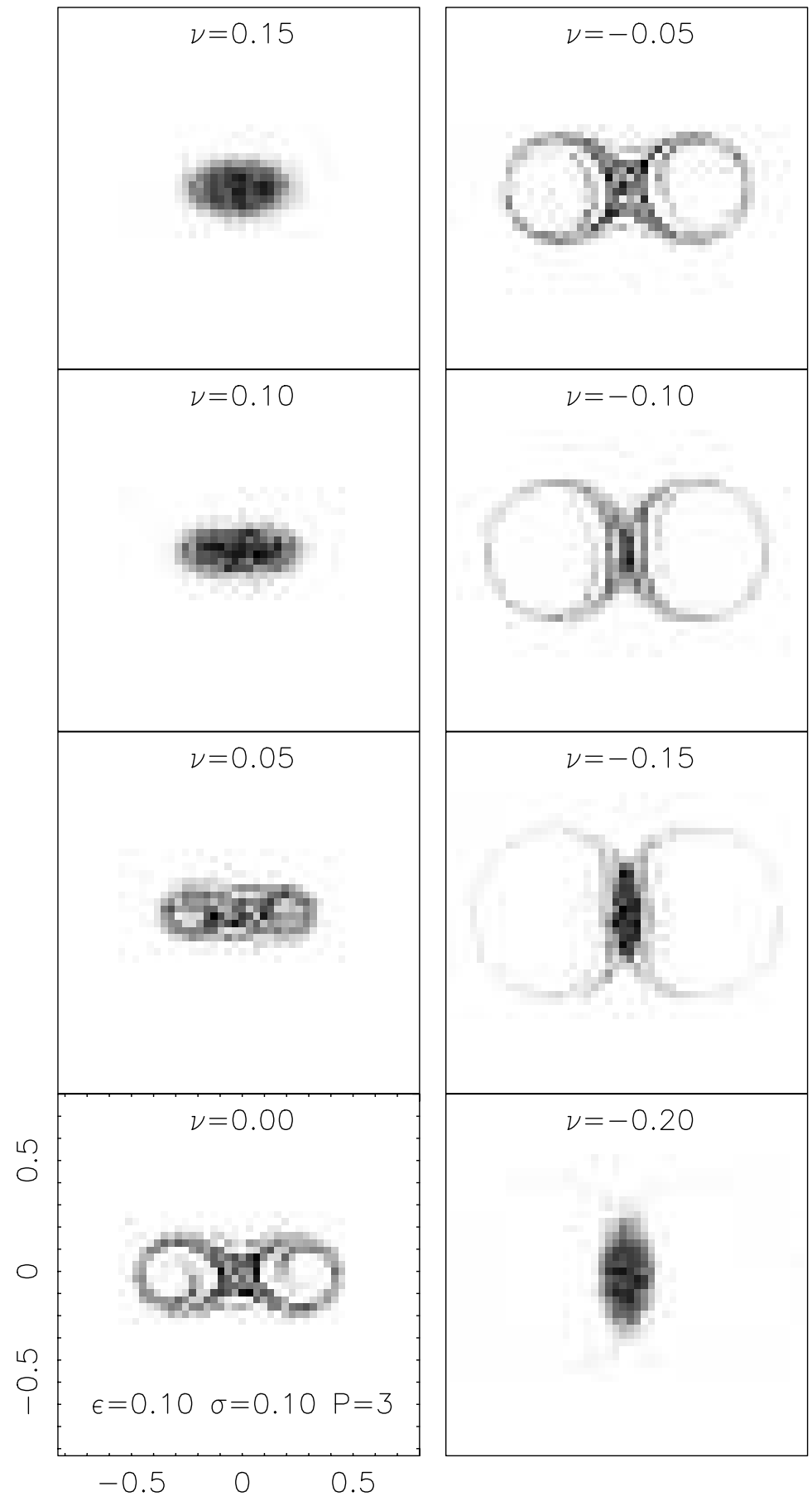

FIG. 3.-Particle distribution following growth of a perturbation with $\bar{\epsilon}=0.1$ for different values of $\bar{\nu}$. The initial dispersion in $j_{3}$ was $\sigma=0.1$. The perturbation was grown in $P=3$ periods, and then the integration continued for 5 times longer than the growth period, so that orbits become more evenly distributed in phase. The $x$ and $y$ axes are defined as in Fig. 1 .

One way to probe the formation timescale of the boxy/ peanut is to search for barred galaxies that have recently formed bars. If boxy/peanuts require many rotation periods to form, then these barred galaxies should have normal (nonboxy) bulges. Because of the high rate of star formation along the bar, Quillen et al. (1997) inferred that NGC 7582 was a case of a recently formed bar. They pointed out that the galaxy bulge did show a strong prominent peanut and so argued that boxy/peanut shaped bulges are formed quickly after the onset of a bar. Observations of edge-on galaxies are therefore consistent with a rapid boxy/peanut formation mechanism such as we have proposed here.

Our scenario does not require the galaxy to buckle in order to form the boxy/peanut shaped bulge. The fire-hose instability scenario for bar buckling (beautifully illustrated in three-dimensional $\mathrm{N}$-body simulations by Raha et al. 1991) predicts that the galaxy will literally be bent in a $U$ shape while the galaxy buckles, but no examples of $U$-shaped edge-on galaxies have been identified in galaxy surveys (with the exception of galaxies that are believed to 




FIG. 4. - (a) Similar to Fig. 3, except the initial dispersion in $j_{3}$ is $\sigma=0.05$. (b) Similar to (a), except the perturbation was grown in 10 rotation periods.

be ram pressure stripped in clusters). It is likely that the period of time during which the galaxy is $U$-shaped is short. However, galaxy collisions have a similar estimated lifetime and are detected in nearby galaxy surveys. We note that Friedli \& Pfenniger (1990) reported that $N$-body simulations with fixed $z$-symmetry did grow boxy/peanut shaped bulges, although at a slower rate than unconstrained simulations.

We have constructed an analytical framework that can be used to predict the height distribution of the peanut as a function of radius and the azimuthal angle in the galaxy plane. The nature of the dependence is primarily determined by the resonance responsible for the vertical excitations. A three-dimensional study of a nearby boxy bulge (such as that seen in our galaxy) could determine which resonance is most likely to be responsible. The shape of the boxy/peanut shaped bulge is also sensitive to the dependence of the gravitational potential on height from the midplane and on the way the bar grew. Further study of boxy/peanut shaped bulges may allow us to place constraints on the vertical shape of the galaxy and on the early evolution of the bar. 


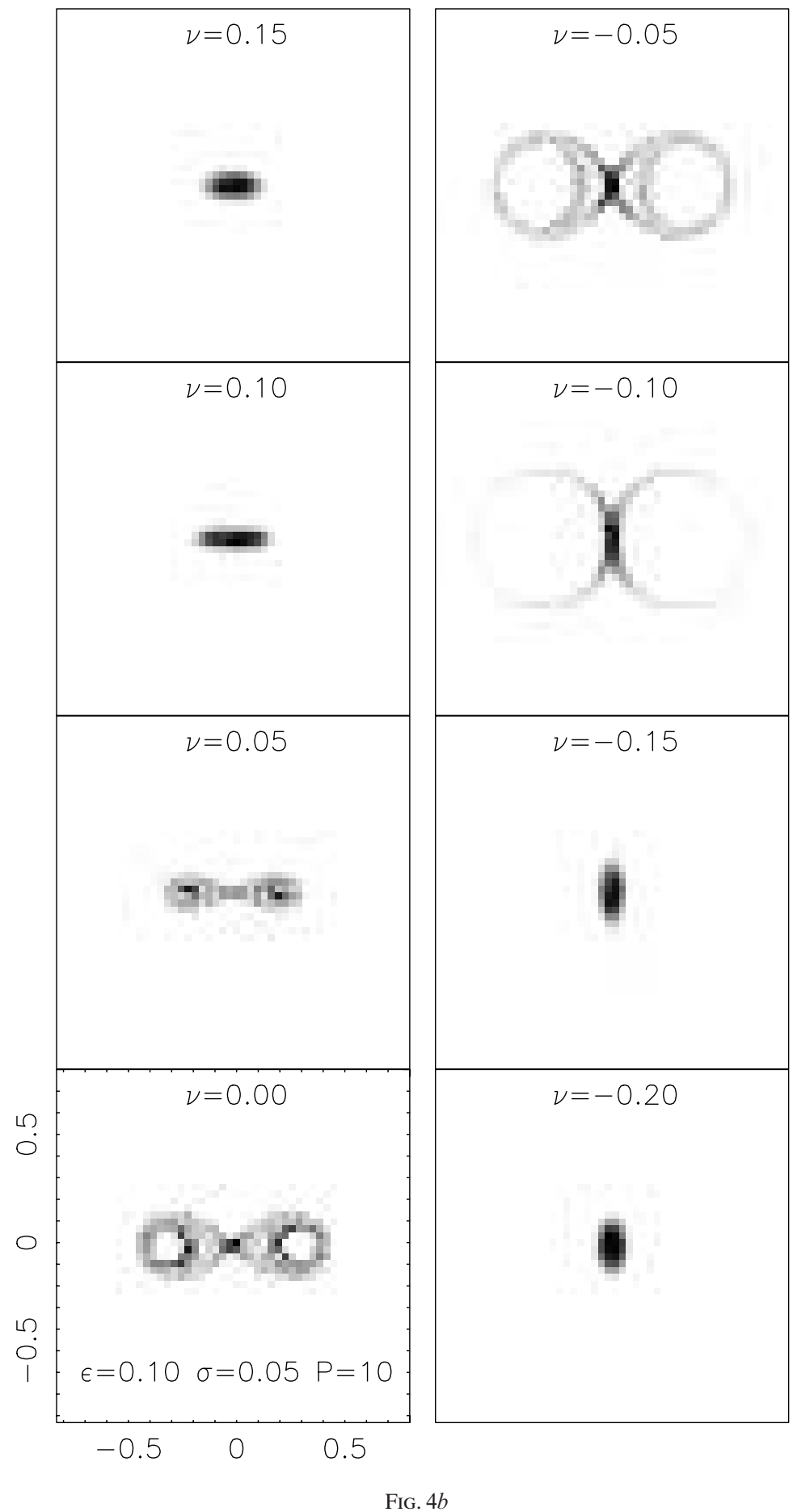

The toy model introduced in this paper was designed to illustrate the process of resonance capture during the growth of a bar. We have argued that the growth of the bar itself causes stars to become trapped into vertical resonances and so lifted out of the plane of the galaxy. However, simultaneous growth of a bar and boxy/peanut shaped bulge has not yet been observed in three-dimensional $N$-body simulations of nonbending bars. This could, in part, be because $N$-body simulations are typically thicker than thin bars such as NGC 7582. Moreover, $N$-body simulations (Pfenniger \& Friedli 1991; Friedli \& Pfenniger 1990; Combes et al. 1990) have shown that the boxy/peanut continues to grow well after the bar is stable. Since resonance capture is likely to be a natural consequence of bar growth, it might be useful to reexamine $N$-body simulations and verify if orbits are trapped into vertical resonances during the bar growth period. After the bar stabilizes, the analytical framework developed here might also be applied toward predicting the evolution of stellar orbits caused by secular evolution of a bar. For example, a slow decrease in pattern speed will also trap particles in the vertical resonances and cause them to be lifted into higher orbits. In this case, $\bar{\nu}$ decreases with time, so that phase space moves downward in Figure 1, causing similar phenomenology as growing the bar; par- 


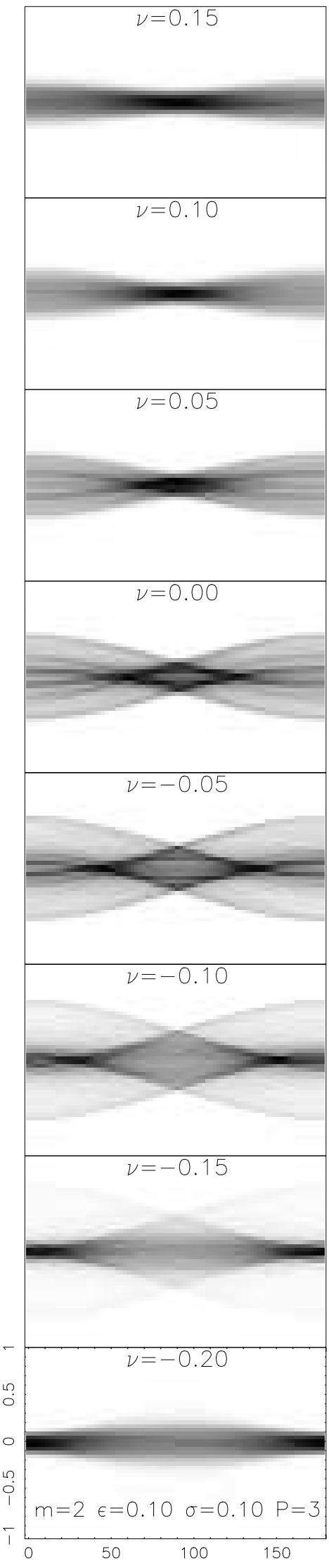

FIG. $5 a$

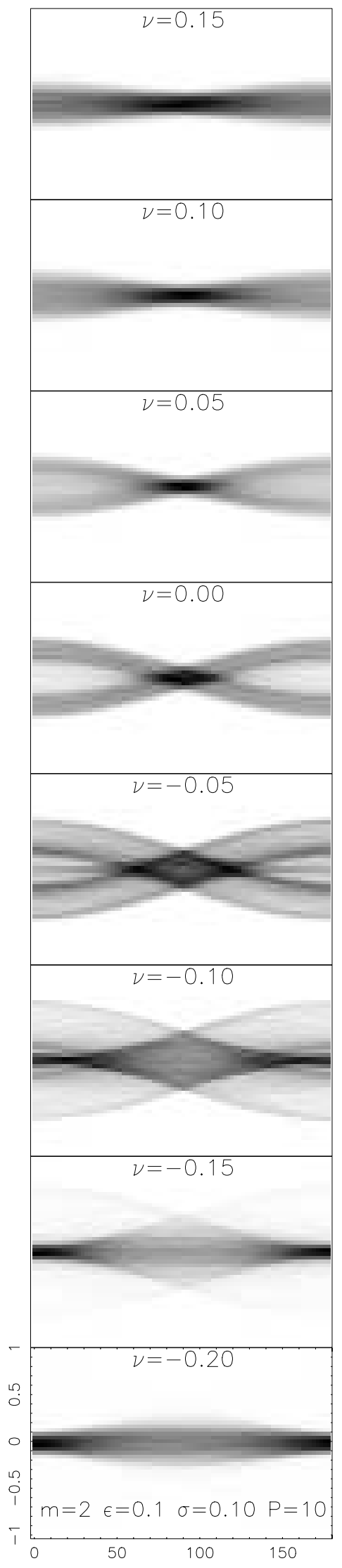

FIG. $5 b$

FIG. 5.- (a) For the distribution in action angle variables displayed in Fig. 3, this shows the vertical distribution as a function of azimuthal angle for the case $m=2$ corresponding to the $1: 1$ resonance. The $x$-axis shows the azimuthal angle, $\theta-\Omega_{b} t$, in the plane of the galaxy in the frame that rotates with the bar and is given in degrees. The $y$-axis shows the $z$ distribution in units of $r_{\text {res. }}$ (b) Same as $(a)$, but for $P=10$ (also shown in Fig. 4b). We see from these figures that we expect a maximum peanut width at the radius where $\bar{\nu} / \bar{\epsilon}=-1$. 



FIg. 6.-Same as Figs. $5 a$ and $5 b$, but for the case when $m=4$ 


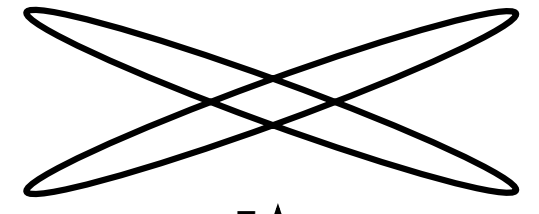

Z

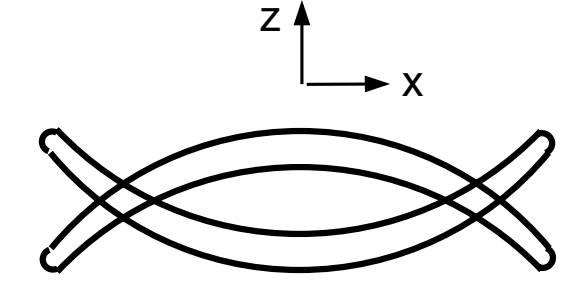

FIG. 7.-Anomalous orbits at the $1: 1$ resonance are shown on the top and give increased widths at the ends of the bar. Banana-shaped orbits at the $2: 1$ resonance are shown on the bottom and give increased widths at the ends and sides of the bar. The $x$-axis is assumed to lie along the major axis of the bar.

ticles trapped into resonances can be lifted higher as the bar evolves.

This work would not have been carried out without helpful discussions with Andrew Anissi and Larry Helfer. A. C. Q. gratefully thanks the Technion for hospitality and support during the fall of 2001 .

\section{APPENDIX}

To put the Hamiltonian into action angle variables to first order, we use the generating function

$$
F_{1}\left(z, \theta^{\prime}\right)=-(\nu / 2) z^{2} \tan \theta^{\prime} .
$$

Following this canonical transformation, the $z$ part of the Hamiltonian restricted to $r=r_{c}$,

$$
H_{z}\left(p_{z} ; z\right)=\frac{1}{2}\left(p_{z}^{2}+\nu^{2} z^{2}\right)+\frac{\lambda z^{4}}{4 !},
$$

becomes

$$
H_{z}\left(I^{\prime} ; \theta^{\prime}\right)=\nu I_{3}^{\prime}+\left(\lambda I^{\prime 2} / 6 \nu^{2}\right) \cos ^{4}\left(\theta^{\prime}\right) .
$$

To put the Hamiltonian into a form that does not depend on the angle, we must do another canonical transformation. Note that $\cos ^{4} \theta=\frac{1}{8} \cos 4 \theta+\frac{1}{2} \cos 2 \theta+\frac{3}{8}$.

We try the following generating function:

$$
F_{2}\left(\theta^{\prime}, I_{3}\right)=I_{3}-\frac{\lambda}{6 \nu^{2}} I_{3}^{2}\left(\frac{1}{4} \sin 2 \theta^{\prime}+\frac{1}{32} \sin 4 \theta^{\prime}\right),
$$

which leads to new variables $I_{3}$ and $\theta_{3}$, such that

$$
\begin{aligned}
& I^{\prime}=I_{3}+\frac{\lambda}{6 \nu} I_{3}^{2}\left(\frac{1}{2} \cos 2 \theta^{\prime}+\frac{1}{8} \sin 4 \theta^{\prime}\right), \\
& \theta_{3}=\theta^{\prime}-\frac{\lambda}{6 \nu^{2}} I_{3}^{\prime}\left(\frac{1}{4} \sin 2 \theta^{\prime}+\frac{1}{32} \sin 4 \theta^{\prime}\right) .
\end{aligned}
$$

To fourth order in $I_{3}^{1 / 2}$, the Hamiltonian becomes

$$
H_{z}\left(I_{3} ; \theta_{3}\right)=\nu I_{3}+\left(\lambda I_{3}^{2} / 32 \nu^{2}\right),
$$

which is independent of $\theta_{3}$, so that $I_{3}$ is a conserved quantity, and we find that we have successfully transferred the Hamiltonian into action angle variables.

Via a similar canonical transformation, the term proportional to $\left(r-r_{c}\right) z^{2}$ in equation (5) disappears, but the term proportional to $\left(r-r_{c}\right)^{2} z^{2}$ remains, yielding the additional term $\left(\kappa^{2}-3 \Omega^{2}\right)\left(\nu I_{1} I_{3} / 4 \kappa\right)$, which we have included in equation (7).

\section{REFERENCES}

Athanassoula, E. 1990, in Galactic Models, ed. J. R. Buchler, S. T. Gottesman, \& J. H. Hunter (Ann. NY Acad. Sci. No. 596) (New York: NY Acad. Sci.), 181

Bettoni, D., \& Galletta, G. 1994, A\&A, 281, 1

Binney, J. J., \& Petrou, M. 1985, MNRAS, 214, 449

Borderies, N., \& Goldreich, P. 1984, Celest. Mech., 32, 127

Burbridge, E. M., \& Burbridge, G. R. 1959, ApJ, 130, 20

Bureau, M., \& Freeman, K. C. 1999, AJ, 118,126

Combes, F., Debbasch, F., Friedli, D., \& Pfenniger, D. 1990, A\&A, 233, 82 Contopoulos, G. 1975, ApJ, 201, 566

de Vaucouleurs, G. 1974, in IAU Symp. 58, Formation and Dynamics of Galaxies, ed., J. R. Shakeshaft (Dordrecht: Reidel), 335

Fridman, A. M., \& Polyachenko, V. L. 1984, Physics of Gravitating Systems (New York: Springer)

Friedli, D., \& Pfenniger, D. 1990, in ESO/CTIO Workshop on Bulges of Galaxies, ed. B. Jarvis \& D. M. Terndrup (Garching: ESO), 265

Henrard, J. 1982, Celest. Mech., 27, 3

Lütticke, R., Dettmar, R.-J., \& Pohlen, M. 2000, A\&A, 362, 435
Merrifield, M. R., \& Kuijken, K. 1999, A\&A, 345, L47

Merritt, D., \& Sellwood, J. A. 1994, ApJ, 425, 551

Murray, C. D., \& Dermott, S. F. 1999, Solar System Dynamics (Cambridge: Cambridge Univ. Press)

Pfenniger, D. 1984, A\&A, 134, 373

. 1985, A\&A, 150, 112

Pfenniger, D., \& Friedli, D. 1991, A\&A, 252, 75

Pfenniger, D., \& Norman, C. 1990, ApJ, 363, 391

Quillen, A. C., Frogel, J. A., \& Gonzalez, R. A. 1994, ApJ, 437, 162

Quillen, A. C., Kuchinski, L. E., Frogel, J. A., \& DePoy, D. L. 1997, ApJ, 481,179

Raha, N., Sellwood, J. A., James, R. A., \& Kahn, F. D. 1991, Nature, 352, 411

Sellwood, J. A., \& Wilkinson, A. 1993, Rep. Prog. Phys., 56, 173

Sridhar, S., \& Touma, J. 1996, MNRAS, 279, 1263

Toomre, A. 1966, in Notes on the 1966 Summer Study Program in Geophysical Fluid Dynamics, Vol. 1 (Woods Hole: Woods Hole Oceanographic Inst.), 111 